Volume 2

\title{
Triple Aim for Clinical Teachers (TACT): Faculty Physician Perceptions on Their Ability to Balance Clinical Quality, Trainee Learning, and Teaching Efficiency
}

Minuja Muralidharan

Anne Getzin

Kjersti E. Knox

Bonnie L. Bobot

Marie M. Forgie

Nicole P. Salvo

Deborah Simpson

Follow this and additional works at: https://aah.org/jpcrr

Part of the Internal Medicine Commons, and the Medical Education Commons

\section{Recommended Citation}

Muralidharan M, Getzin A, Knox KE, Bobot BL, Forgie MM, Salvo NP, Simpson D. Triple Aim for Clinical Teachers (TACT): Faculty Physician Perceptions on Their Ability to Balance Clinical Quality, Trainee Learning, and Teaching Efficiency. J Patient Cent Res Rev 2015;2:209-210. http://dx.doi.org/10.17294/ 2330-0698.1233

Published quarterly by Midwest-based health system Advocate Aurora Health and indexed in PubMed Central, the Journal of Patient-Centered Research and Reviews (JPCRR) is an open access, peer-reviewed medical journal focused on disseminating scholarly works devoted to improving patient-centered care practices, health outcomes, and the patient experience. 
and subsequent PCI to determine if there is increased risk of stent thrombosis in specific post-CABG coronary artery territories and if these altered overall cardiovascular outcomes.

Methods: A retrospective review of our database on all patients presenting with stent thrombosis over the last 5 years was performed. Patients were included based on the accepted Academic Research Consortium definition of stent thrombosis.

Results: From January 2009 to February 2014, 220 patients were found to have had a stent thrombosis. Of these, 110 $(50.0 \%)$ had left anterior descending (LAD) artery lesions, $82(37.3 \%)$ had right coronary artery (RCA) lesions and $26(11.8 \%)$ had a stent thrombosis in the left circumflex artery (LCx). Prevalences of traditional risk factors were essentially equivalent regardless of which coronary artery developed stent thrombosis. All patients were on dual antiplatelet prior to developing stent thrombosis. Further analysis revealed $38(17.3 \%)$ had a prior history of CABG. A significant difference among the location of stent thrombosis and the history of CABG $(\mathrm{P}=0.043)$ was seen; $30.8 \%(\mathrm{n}=8)$ of patients with $\mathrm{LCx}$ stent thrombosis had prior $\mathrm{CABG}$ compared to $10.9 \%(n=12)$ and $22 \%(n=18)$ with LAD and RCA stent thrombosis, respectively.

Conclusion: In a large cohort of patients with stent thrombosis, LAD and RCA lesions were predominant, with LAD lesions representing half of all stent thromboses. PCI of these coronary territories thus infers a higher risk of stent thrombosis even in the presence of optimal medical therapy. Once stent thrombosis occurs, no significant difference in outcomes is seen based on location of the lesion alone. Additionally, patients who had prior CABG were significantly more likely to have stent thrombosis in the LCx and less likely in the LAD. This could be due to the fact that the left internal mammary artery graft is more often patent than vein grafts, which are more often anastomosed to the LCx and RCA and are at higher risk of needing stent placement after CABG.

\section{In Vitro Growth Suppression of Renal Carcinoma Cells by Curcumin}

Santhi D. Konduri, Madhavi Latha Yadav Bangaru, Phu Thanh Do, Shenglin Chen, Jeffrey Woodliff, Sanjay Kansra

\section{Aurora Research Institute, Aurora Health Care; Medical College of Wisconsin}

Background: Malignant clear cell renal carcinoma (ccRCC) is an aggressive tumor that is highly resistant to chemotherapy and radiation. Current therapeutic approaches to management of ccRCC have not significantly improved patient survival, therefore novel therapies are needed. The von Hippel-Lindau tumor suppressor gene is frequently mutated in ccRCC resulting in unregulated transcriptional activity of hypoxia-inducible factors (HIF) $1 \alpha$ and $2 \alpha$. HIFmediated transcription leads to increased growth factor expression and growth factor receptor (GFR)-mediated signaling. NFKB and STAT3 are phosphorylated in response to GFR activation and modulate gene expression, which promotes cell growth and invasion. Activated $\mathrm{NF \kappa B}$ and STAT3 expression is associated with ccRCC pathogenesis.

Purpose: The dietary polyphenol curcumin is a welldocumented antitumor agent and a known inhibitor of $\mathrm{NF} \kappa \mathrm{B}$ and STAT3 activation. Given the lack of effective therapies that block ccRCC progression, our objective was to examine whether curcumin could suppress the growth and migration of ccRCC cells, and whether this suppression was mediated via inhibition of NFKB and STAT3 activity.

Methods: Human ccRCC cell lines (769-p, 786-o, Caki-1, ACHN and A-498 cells) were exposed to curcumin to assess the impact of curcumin on ccRCC cell viability. To examine the mechanism by which curcumin induced cell death, we used 769-p cells, a highly aggressive human ccRCC cell line that does not express functional von Hippel-Lindau protein. The impact of curcumin on the phosphorylation status and transcriptional activity of NFKB and STAT3, in 769-p cells, was determined.

Results: Our results show that in ccRCC cells curcumin decreased cell proliferation and cell viability, abolished clonogenic property, induced apoptosis and blocked cellular migration. The growth suppressive and proapoptotic effects of curcumin were accompanied by decreased phosphorylation and transcriptional activity of NFאB and STAT3.

Conclusion: The ability of curcumin to induce apoptosis and inhibit migration of ccRCC cells justifies additional studies that explore the potential of developing curcumin or other $\mathrm{NF} \kappa \mathrm{B}$ and STAT3 inhibitors as novel therapeutic agents in the management of ccRCC.

\section{Triple Aim for Clinical Teachers (TACT): Faculty Physician Perceptions on Their Ability to Balance Clinical Quality, Trainee Learning, and Teaching Efficiency}

Minuja Muralidharan, Anne Getzin, Kjersti E. Knox, Bonnie L. Bobot, Marie M. Forgie, Nicole P. Salvo, Deborah Simpson

Departments of Internal Medicine, Family Medicine, Obstetrics and Gynecology, and Academic Affairs, Aurora UW Medical Group

Background: A common challenge facing teaching physicians is balancing high-quality student and resident teaching with efficient, high-quality care and patient service. Publicly accessible clinic performance reports increasingly affect where patients seek care and demand that teaching clinics rise to consumer expectations while training future physicians to function in the modern health care workplace. Limited information is available to guide physicians to achieve the triple aim for clinical teachers (TACT): clinical quality/patient experience, trainee learning, and teaching efficiency.

Purpose: To understand clinical teachers' TACT-related 
experiences, perceptions and preferences for how to learn TACT-associated skill sets to improve their competence as teachers.

Methods: A 7-question needs assessment survey was distributed to teaching faculty members in family medicine, internal medicine and ob/gyn in a health care system. Ranking, rating and free-response item formats were used to determine teachers' prioritization of care management and patient satisfaction metrics within medical education and their perceived skills and limitations in incorporating these factors into medical education. Data was analyzed using descriptive statistics and narrative comments using qualitative thematic analysis. This project was deemed "not human subjects research" by Aurora Health Care.

Results: A 78\% response rate was obtained (32/41). Respondents' top 3 teaching priorities were "Meeting specific clerkship objectives/residency milestones," "Impact on your time/teaching efficiency" and "Service quality priorities for the clinic." Respondents ranked learner's evaluation of teaching among their lowest priorities. $63 \%$ of respondents reported that they involve learners in improvement efforts (quality, safety, patient experience). Respondents identified a variety of strategies for involving learners in improvement efforts (medical students initiate patient callback, follow up on lab tests, check/address health maintenance items; residents identify a care management target), although time was consistently identified as a barrier to learner involvement.

Conclusion: Survey results confirmed that clinical teachers place value on integrating efforts to enhance clinical quality/ patient experience as they teach yet face challenges to TACT goal attainment. Findings will inform description of successful TACT strategies, assessment of their effectiveness and faculty development initiatives.

\section{Reducing Readmission Rates in Acute Pancreatitis Through Patient Education and Risk Assessment}

\section{Jordan T. Vulcano}

\section{Department of Internal Medicine, Aurora Sinai Medical Center}

Background: Early hospital readmissions are a direct burden on both our patients' well-being and health care system as a whole. Acute pancreatitis is a top offender, with countless 30-day readmissions. Studies have showed a consistently higher than average 30-day readmission rates in acute pancreatitis, around $19 \%$. This is significantly higher than the average all-cause readmission rate at Aurora Health Care hospitals. This quality improvement project aimed to reduce the rate of acute pancreatitis 30-day readmission rates at several Aurora hospitals through patient education and a readmission risk assessment tool.

Purpose: To clarify some of the risk factors associated with acute pancreatitis readmissions and reduce 30-day acute pancreatitis readmission rates through patient education and risk assessment to facilitate a safe discharge.
Methods: Project was conducted out of Aurora's Sinai, St. Luke's and West Allis Medical Centers with a total of 18 patients with acute pancreatitis admitted predominantly to the internal medicine teaching service between February 2014 and October 2014. Patients were seen within 1-2 days of admission and provided one-on-one education with a handout on acute pancreatitis. In addition, a 30-day pancreatitis readmission predictor (PRP) score was used to classify patient as low (5\%), moderate $(17 \%)$ or high $(68 \%)$ risk for readmission via Epic health record's "Dot Phrase." Subsequent readmissions, 14-day follow-up, total hospitalizations and emergency department visits were tracked through present. This was compared to readmission rates of a randomly selected control group of 18 patients admitted with acute pancreatitis.

Results: Patients had PRP scores ranging from 0 to 4 , with an average of 1 (rounded from 0.78). Of the 18 patients in the study group, only 2 were readmitted within 30 days for pancreatitis, or $11.1 \%$. The control group had 3 (16.7\%) readmissions within 30 days. Patients with alcohol-related pancreatitis were more likely to have a higher PRP (1.0) and readmission rate $(20 \%, 2 / 10)$.

Conclusion: A diagnosis of acute pancreatitis places the patient at a significantly higher than average risk of readmission. This project was able to reduce readmission rates from $16.7 \%$ to $11.1 \%$ by simple patient education and readmission risk assessment. Readmissions are detrimental to both the patient and health care system. This project serves as a starting point for reducing readmissions not only in acute pancreatitis patients but potentially other diagnosisspecific readmission initiatives.

\section{Tertiary Center Experience of Catheter-Directed Thrombolysis for Immediately Threatened Acute Lower Limb Ischemia of Native Vessels and Bypass Graft Thrombosis}

Hani Hashim, M. Fuad Jan, Maharaj Singh, Suhail Allaqaband, Tanvir Bajwa, Anjan Gupta

\section{Aurora Cardiovascular Services, Aurora Health Care; Aurora Research Institute, Aurora Health Care}

Background: Catheter-directed thrombolysis (CDT) is an effective therapy and a class I indication for patients with acute limb ischemia (ALI, Rutherford categories I and IIa) of less than 14 days duration, and class IIb indication for ALI (Rutherford category IIb) with symptoms more than 14 days duration. However, there is no consensus on the initial management option for ALI (Rutherford category IIb) with symptoms less than 14 days duration.

Purpose: To evaluate the safety, efficacy and outcome of CDT, with or without bailout Angiojet mechanical thrombectomy, in patients with immediately threatened acute lower extremity ischemia (Rutherford category IIb) as a minimally invasive alternative to emergent surgical revascularization.

Methods: We retrospectively reviewed data on 69 